\title{
Adult woman with giant thrombosed multiple coronary aneurysms
}

\author{
Javier Castrodeza ${ }^{1}$, Ana Revilla ${ }^{1,2}$, Gregorio Laguna ${ }^{3}$, \\ Ignacio J. Amat-Santos ${ }^{1,2}$, Teresa Sevilla ${ }^{1,2}$ \\ ${ }^{1}$ Department of Cardiology, ICICOR, Hospital Clínico Universitario, Valladolid, Spain \\ ${ }^{2}$ CIBER de Enfermedades Cardiovasculares (CIBERCV), Valladolid, Spain \\ ${ }^{3}$ Department of Cardiac Surgery, Hospital Clínico Universitario, Valladolid, Spain
}

A 55-year-old Caucasian woman with history of hypertension, dyslipidemia, and no relevant disease in her childhood was referred for catheterization due to a non-ST-elevation myocardial infarction. A transthoracic echocardiogram showed a rounded structure with hyperechogenic limits anterior to the auriculo-ventricular sulcus in the two-chamber view (Fig. 1A). The angiogram revealed a three-vessel disease with an aneurysm of the proximal left anterior descendent artery with severe stenosis, a significant stenosis followed by another aneurysm in the proximal circumflex (Fig. 1C) and a completely thrombosed aneurysm in the proximal right coronary (Suppl. Video 1 and 2). A multidetector cardiac computed tomography scan was then performed and revealed a calcified and partially thrombosed aneurysm of $53 \mathrm{~mm}$ with a diameter of $29 \times 24 \mathrm{~mm}$ in the proximal
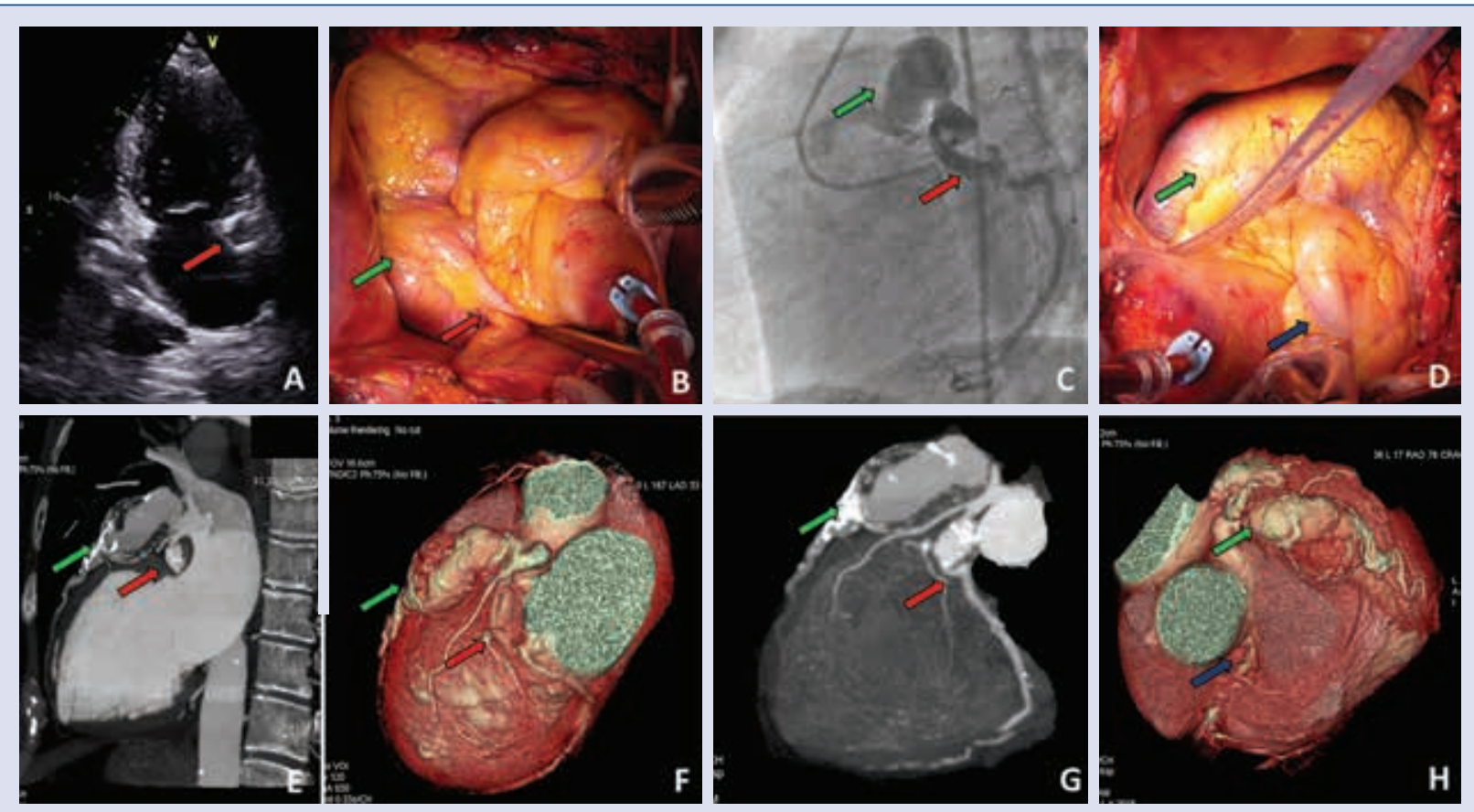

Figure 1. A-H. Image matching of computed tomography scan, angiography, two-chamber view, and surgical view.

Address for correspondence: Ana Revilla, MD, PhD, ICICOR, Hospital Clínico Universitario de Valladolid,

C/Ramón y Cajal 3, Valladolid, Spain, tel: +34 9834200 26, fax: +34 9832553 05, e-mail: arevillaorodea@gmail.com

Received: 08.01.2018 Accepted: 16.01 .2018 
left anterior descendent (green arrow), another of $17 \times 18 \mathrm{~mm}$ in the proximal circumflex (red arrow) and a calcified and thrombosed proximal right coronary aneurysm (blue arrow) with appropriate distal vessels (Fig. 1E-H). Supra-aortic trunks were normal. She underwent surgery for coronary artery by-pass grafting (Fig. 1B, D; Suppl. Video 3), as stenosis was the predominant feature, using both internal mammary arteries and a saphenous vein. She was discharged with no incidence.
Giant and multiple coronary aneurysms are uncommon in adulthood. Possible etiologies are atherosclerosis, Kawasaki and Takayasu disease and some systemic and infective disorders. Multidetector cardiac computed tomography scan is an excellent technique to plan the surgical intervention as it allows a complete characterization and evaluation of the aneurysms and distal vessels.

Conflict of interest: None declared 